PaWeŁ BŁażeJ (Wrocław)

\title{
ROBUST ESTIMATION OF THE SCALE AND WEIGHTED DISTRIBUTIONS
}

Abstract. The concept of robustness given by Zieliński (1977) is considered in cases where violations of models are generated by weight functions. Uniformly most bias-robust estimates of the scale parameter, based on order statistics, are obtained for some statistical models. Extensions of results of Zieliński (1983) and Bartoszewicz (1986) are given.

1. Preliminaries. Let $X$ and $Y$ be random variables with absolutely continuous distributions $F$ and $G$ respectively and $F(0)=G(0)=0$. Denote by $S_{F}$ the support and by $F^{-1}(u)=\inf \{x: F(x) \geq u\}, u \in(0,1)$, the quantile (or reversed) function of $F$, and similarly for $G$.

We recall some basic facts about stochastic orders. We say that a random variable $X$ is smaller than $Y$ in the stochastic order $\left(F \leq_{\text {st }} G\right)$ if $F(x) \geq G(x)$ for every $x$.

We say that $X$ is smaller than $Y$ in the star order $\left(F \leq_{*} G\right)$ if $G^{-1} F(x) / x$ is non-decreasing on $S_{F}$ where $G^{-1} F$ denotes the composition of $G^{-1}$ and $F$.

We say that $X$ is smaller than $Y$ in the convex order $\left(F \leq_{\mathrm{c}} G\right)$ if $G^{-1} F(x)$ is convex on $S_{F}$. It is well known that the convex order implies the star order.

A distribution $F$ is said to be IFR (increasing failure rate) if $F \leq_{\mathrm{c}} G$ where $G$ is an exponential distribution. Let $X_{1: n}, \ldots, X_{n: n}$ be order statistics in a sample $X_{1}, \ldots, X_{n}$ from the distribution $F$ and let $F_{i: n}$ denote the distribution of $X_{i: n}, i=1, \ldots, n ; G_{i: n}$ is defined similarly. It is well known that if $F \leq_{\mathrm{st}} G$ then $F_{i: n} \leq_{\mathrm{st}} G_{i: n}$; also if $F \leq_{\mathrm{c}} G$ (resp. $F \leq_{*} G$ ) we obtain $F_{i: n} \leq_{\mathrm{c}} G_{i: n}\left(\right.$ resp. $\left.F_{i: n} \leq_{*} G_{i: n}\right)$ for any $i$.

We will use the following lemma:

2000 Mathematics Subject Classification: 60E15, 60E07, 62 N05.

Key words and phrases: weighted distribution, IFR, DFR, partial orders of distributions.

Research supported by KBN Grant N20104631/3733. 
Lemma 1 (Barlow and Proschan, 1966). If $F \leq_{*} G$ then $E\left[X_{i: n}\right] / E\left[Y_{i: n}\right]$ is a non-decreasing function of $i=1, \ldots, n$.

Let $F$ be a distribution function, and $w: \mathbb{R} \rightarrow \mathbb{R}^{+}$such that $0<$ $E[w(X)]<\infty$. Then

$$
F_{w}(x)=\frac{1}{E[w(X)]} \int_{0}^{x} w(z) d F(z)
$$

is the weighted distribution associated with $F$ with weight function $w$. The weighted distribution $F_{w}$ has a density

$$
f_{w}(x)=\frac{w(x) f(x)}{E[w(X)]}
$$

The idea of weighted distributions is due to Fisher (1934). Rao (1965) defined weighted distributions with a general weight function $w$. Rao and Patil (1977) provided some statistical models leading to weighted distributions and applied their results to the analysis of data relating to human population and ecology. Patil and Ord (1976) defined some classes of distributions which are invariant under weighting with weight functions of type $x^{\alpha}, \alpha>0$. Bayarri and Berger (1998) and also Chung and Kim (2004) considered robustness for weighted distributions from the Bayesian point of view.

Many authors, e.g. Jain et al. (1989) and Bartoszewicz and Skolimowska (2006), studied preservation of classes of life distributions under weighting. The following result will be used.

LEMMA 2 (Jain et al., 1989). If $F$ is IFR, and $w$ is increasing and concave, then $F_{w}$ is IFR.

2. Introduction. Zieliński (1977) proposed the following concept of robustness. Let the original statistical model be $M_{0}=\left(\mathcal{X}, \mathcal{A}, \mathcal{P}_{0}\right)$ where $(\mathcal{X}, \mathcal{A})$ is a given measurable space and $\mathcal{P}_{0}$ is a given subset of the class $\mathcal{P}$ of all probability measures. Let $\pi: \mathcal{P}_{0} \rightarrow 2^{\mathcal{P}}$ be a function called a violation of $M_{0}$ which has the property: $P \in \pi(P)$ for all $P \in \mathcal{P}_{0}$. Define $\mathcal{P}_{1}=\bigcup_{P \in \mathcal{P}_{0}} \pi(P)$. Thus $M_{1}=\left(\mathcal{X}, \mathcal{A}, \mathcal{P}_{1}\right)$ is an extension of the model $M_{0}$. Let $T$ be a suitable statistic with distribution $P^{T}(\cdot)=P\left(T^{-1}(\cdot)\right)$ and $\varrho$ be a real-valued function on $\mathcal{P}_{1}$. We have the following definitions.

Definition 1 (Zieliński, 1977). The function $r_{T}: \mathcal{P}_{0} \rightarrow \mathbb{R}^{+}$defined as

$$
r_{T}(P)=\sup \left\{\varrho\left(Q^{T}\right): Q \in \pi(P)\right\}-\inf \left\{\varrho\left(Q^{T}\right): Q \in \pi(P)\right\}
$$

is called the $\varrho$-robustness of the statistic $T$ in $M_{1}$.

Definition 2 (Zieliński, 1977). A statistic $T^{0}$ is uniformly most @-robust in a given class $\mathcal{T}$ of statistics if

for every $P \in \mathcal{P}_{0}$ and $T \in \mathcal{T}$.

$$
r_{T^{0}}(P) \leq r_{T}(P)
$$


Zieliński (1983) and Bartoszewicz (1986) used the above definitions to solve the problem of robustness of unbiased estimation of the scale parameter in some models.

EXAmple 1 (Bartoszewicz, 1986). Consider the statistical model

$$
M_{0}=\left(\mathbb{R}^{+}, \mathcal{B}^{+},\{F(\cdot ; \theta): \theta>0\}\right),
$$

where $\{F(\cdot ; \theta): \theta>0\}$ is a class of distributions with scale parameter $\theta$ (i.e. $F(x ; \theta)=F(x / \theta))$.

For fixed continuous distributions $H, K$, where $H(0)=K(0)=0$ and $H \leq_{\text {st }} K$, for every $\theta$ we define $\pi_{H, K}(\theta)$ to be any set of distributions $G(\cdot ; \theta)$ satisfying the following conditions:

(i) $H(\cdot ; \theta) \leq_{\text {st }} G(\cdot ; \theta) \leq_{\text {st }} K(\cdot ; \theta)$ for every $G(\cdot ; \theta) \in \pi_{H, K}(\theta)$, where $H(x ; \theta)=H(x / \theta), K(x ; \theta)=K(x / \theta) ;$

(ii) $H(\cdot ; \theta), K(\cdot ; \theta) \in \pi_{H, K}(\theta)$;

(iii) $\pi_{H, K}\left(\theta^{\prime}\right) \cap\{F(\cdot ; \theta): \theta>0\}=\left\{F\left(\cdot ; \theta^{\prime}\right)\right\}$ for every $\theta^{\prime}>0$.

The set $\pi_{H, K}(\theta)$ is called a violation of $M_{0}$ generated by stochastic order. Let $\mathcal{T}$ be the following class of unbiased estimates of the scale parameter $\theta$ based on a sample $X_{1}, \ldots, X_{n}$ :

$$
\mathcal{T}=\left\{T=\sum_{j=1}^{n} a_{j} X_{j: n}: a_{j} \geq 0, j=1, \ldots, n, E_{\theta}[T]=\theta, \theta>0\right\},
$$

where $E_{\theta}[T]$ is the expected value of $T$ if the underlying distribution is $F(\cdot ; \theta)$. The problem is to find the uniformly bias-robust (under the above violation) estimate of $\theta$ in the class $\mathcal{T}$. Bartoszewicz (1986), using Lemma 1 , proved the following theorem.

ThEOREM 1 (Bartoszewicz, 1986). Under the violation $\pi_{H, K}(\theta)$ of the model $M_{0}$ :

(i) if $H(\cdot ; \theta) \leq_{*} F(\cdot ; \theta) \leq_{*} K(\cdot ; \theta)$ then $X_{1: n} / E_{1}\left[X_{1: n}\right]$ is the uniformly most bias-robust estimate of $\theta$ in $\mathcal{T}$;

(ii) if $K(\cdot ; \theta) \leq_{*} F(\cdot ; \theta) \leq_{*} H(\cdot ; \theta)$ then $X_{n: n} / E_{1}\left[X_{n: n}\right]$ is the uniformly most bias-robust estimate of $\theta$ in $\mathcal{T}$.

In this paper we give an extension of Theorem 1 . We consider the violation of the original model $M_{0}$ which is defined by a certain class of weight functions.

3. Results. Let us consider the statistical model

$$
M_{0}=\left(\mathbb{R}^{+}, \mathcal{B}^{+} ;\left\{F(\cdot ; \theta): \theta \in \mathbb{R}^{+}\right\}\right),
$$

where $\mathbb{R}^{+}$is the real positive half-line, $\mathcal{B}^{+}$is the family of Borel subsets of $\mathbb{R}^{+}$and $\left\{F(\cdot ; \theta): \theta \in \mathbb{R}^{+}\right\}$is a family of distributions with scale parameter 
$\theta \in \mathbb{R}^{+}$. This model is violated in such a way that in fact for any $\theta$ we observe the weighted distributions $F_{w}(\cdot ; \theta)$ where $w$ belongs to a suitable class $\mathcal{W}$ of functions.

Next we define the following class of functions. For fixed $a<0$ and $b>0, \mathcal{W}_{a, b}$ is the class of non-negative functions which satisfy the following conditions:

(i) $w(x) / x^{b}$ is non-increasing,

(ii) $w(x) / x^{a}$ is non-decreasing.

It is obvious that the constant functions belong to $\mathcal{W}_{a, b}$. Next we prove the following theorem.

ThEOREM 2. For every distribution $F$ and $w \in \mathcal{W}_{a, b}$ we have

$$
F_{x^{a}} \leq_{\text {st }} F_{w} \leq_{\text {st }} F_{x^{b}}
$$

Proof. The proof is similar to the proof of Theorem 4.1 in Arnold (1980). We will prove only $F_{w} \leq_{\text {st }} F_{x^{b}}$. It suffices to show that

$$
F_{w}(x)-F_{x^{b}}(x) \geq 0 \quad \text { for every } x .
$$

We have

$$
\begin{aligned}
& \frac{1}{E[w(X)]} \int_{0}^{x} w(z) d F(z)-\frac{1}{E\left[X^{b}\right]} \int_{0}^{x} z^{b} d F(z) \\
& =\frac{1}{E[w(X)]} \int_{0}^{F(x)} w\left(F^{-1}(u)\right) d u-\frac{1}{E\left[X^{b}\right]} \int_{0}^{F(x)}\left(F^{-1}(u)\right)^{b} d u \\
& =\frac{1}{E[w(X)]} \int_{0}^{F(x)}\left[w\left(F^{-1}(u)\right)-\left(F^{-1}(u)\right)^{b} \frac{E[w(X)]}{E\left[X^{b}\right]}\right] d u .
\end{aligned}
$$

Since the function $w\left(F^{-1}(u)\right) /\left(F^{-1}(u)\right)^{b}$ is non-increasing in $u$ and

$$
\frac{1}{E[w(X)]} \int_{0}^{1}\left[w\left(F^{-1}(u)\right)-\left(F^{-1}(u)\right)^{b} \frac{E[w(X)]}{E\left[X^{b}\right]}\right] d u=0
$$

the integrand

$$
w\left(F^{-1}(u)\right)-\left(F^{-1}(u)\right)^{b} \frac{E[w(X)]}{E\left[X^{b}\right]}
$$

is first positive then negative as $u$ varies from 0 to 1 . Thus (1) holds. The proof of the inequality $F_{x^{a}} \leq_{\text {st }} F_{w}$ is similar.

Using $\{F(\cdot ; \theta): \theta>0\}$ and $\mathcal{W}_{a, b}$ we can construct the following class of distributions:

$$
\pi_{a, b}(\theta)=\left\{F_{w}(\cdot ; \theta): w \in \mathcal{W}_{a, b}\right\}
$$


It is obvious that for every $\theta>0$ we have $F(\cdot ; \theta) \in \pi_{a, b}(\theta)$. Next we give the following characterization of the family $\left\{\pi_{a, b}(\theta): \theta>0\right\}$.

THEOREM 3. Let $\{F(\cdot ; \theta): \theta>0\}$ be the class of distributions with scale parameter $\theta$ and $f(\cdot ; \theta)$ be a density of $F(\cdot ; \theta)$. If for any $w \in \mathcal{W}_{a, b}$ and $\theta \neq \theta^{\prime}$ the function $w(\cdot) f\left(\cdot ; \theta^{\prime}\right) / f(\cdot ; \theta)$ is not in $\mathcal{W}_{a, b}$, then

$$
\pi_{a, b}\left(\theta^{\prime}\right) \cap\{F(\cdot ; \theta): \theta>0\}=\left\{F\left(\cdot ; \theta^{\prime}\right)\right\}
$$

and

$$
\pi_{a, b}\left(\theta^{\prime}\right) \cap \pi_{a, b}(\theta)=\emptyset .
$$

Proof. For $w(x) \equiv 1$ we obtain $f\left(\cdot ; \theta^{\prime}\right) / f(\cdot ; \theta) \notin \mathcal{W}_{a, b}$, which yields (2). To prove (3) suppose there exist $w_{1}, w_{2}$ such that $\theta \neq \theta^{\prime}$ and $F_{w_{1}}(x ; \theta)=$ $F_{w_{2}}\left(x ; \theta^{\prime}\right)$ for every $x$. Then

$$
\begin{aligned}
F_{w_{1}}\left(x ; \theta^{\prime}\right) & =\frac{1}{E\left[w_{1}(X)\right]} \int_{0}^{x} w_{1}(z) f\left(z ; \theta^{\prime}\right) d z \\
& =\frac{1}{E\left[w_{1}(X)\right]} \int_{0}^{x} w_{1}(z) \frac{f\left(z ; \theta^{\prime}\right)}{f(z ; \theta)} f(z ; \theta) d z .
\end{aligned}
$$

Thus $w_{2}(z)=w_{1}(z) f\left(z ; \theta^{\prime}\right) / f(z ; \theta) \in \mathcal{W}_{a, b}$.

Let $T \in \mathcal{T}$. Denote by $E_{w ; \theta}[T]$ the expected value of $T$ if the underlying distribution is $F_{w}(\cdot ; \theta)$. Obviously $E_{\theta}[T]$ denotes the expected value of $T$ if $w(x)=$ const.

Let

$$
r_{T}(\theta)=\sup _{F_{w}(\cdot ; \theta) \in \pi_{a, b}(\theta)} E_{w ; \theta}[T]-\inf _{F_{w}(\cdot ; \theta) \in \pi_{a, b}(\theta)} E_{w ; \theta}[T]
$$

be the oscillation of the bias of $T$ over $\pi_{a, b}(\theta)$. This function is a measure of robustness of the estimate $T$ with respect to the bias violation. Our problem is to find $T^{0}$ such that $r_{T^{0}}(\theta) \leq r_{T}(\theta)$ for every $\theta$ and $T \in \mathcal{T}$.

It is well known that if $F \leq_{\text {st }} G$ then $E\left[X_{i: n}\right] \leq E\left[Y_{i: n}\right]$ for any $i$. Thus from Theorem 2 we obtain

$$
\inf _{F_{w}(\cdot ; \theta) \in \pi_{a, b}(\theta)} E_{w ; \theta}[T]=E_{x^{a} ; \theta}[T], \quad \sup _{F_{w}(\cdot ; \theta) \in \pi_{a, b}(\theta)} E_{w ; \theta}[T]=E_{x^{b} ; \theta}[T] .
$$

Similarly to Zieliński (1983), the problem of finding $T^{0}$ is reduced to minimizing

$$
\sum_{j=1}^{n} a_{j}\left(E_{x^{b} ; \theta}\left[X_{j: n}\right]-E_{x^{a} ; \theta}\left[X_{j: n}\right]\right) \quad \text { for every } \theta
$$

under the unbiasedness condition

$$
\sum_{j=1}^{n} a_{j} E_{\theta}\left[X_{j: n}\right]=\theta, \quad \theta>0
$$


which is equivalent to

$$
\sum_{j=1}^{n} a_{j} E_{1}\left[X_{j: n}\right]=1
$$

For every $T \in \mathcal{T}$ from (4) we have

$$
r_{T}(\theta)=\sum_{j=1}^{n} a_{j}\left(E_{x^{b} ; \theta}\left[X_{j: n}\right]-E_{x^{a} ; \theta}\left[X_{j: n}\right]\right) \geq \min _{1 \leq j \leq n} \frac{E_{x^{b} ; \theta}\left[X_{j: n}\right]-E_{x^{a} ; \theta}\left[X_{j: n}\right]}{E_{1}\left[X_{j: n}\right]} .
$$

Thus from the fact $F_{x^{\alpha}}(x ; \theta)=F_{x^{\alpha}}(x / \theta ; 1), \alpha>0$, we obtain

$$
T^{0}=\frac{X_{j: n}}{E_{1}\left[X_{j: n}\right]} \quad \text { for some } j=1, \ldots, n \text {. }
$$

REMARK 1. It is not to hard to see that a similar result can be obtained if the weight function depends on the scale parameter $\theta$, i.e. $w_{\theta}(x)=w(x / \theta)$.

4. Robust estimation of the scale parameter in the weighted exponential model. Let the original model be $M_{0}=\left(\mathbb{R}^{+}, \mathcal{B}^{+},\{F(\cdot ; \theta): \theta>0\}\right)$ where $F(\cdot ; \theta)$ is an exponential distribution function with scale parameter $\theta$. We give an extension of $M_{0}$ under weighting. Define

$$
\pi_{a, b}(\theta)=\left\{F_{w}(\cdot ; \theta): w \in \mathcal{W}_{a, b}\right\}, \quad \text { where } \quad-1<a<0, b>0 .
$$

Thus we obtain

$$
M_{1}=\left(\mathbb{R}^{+}, \mathcal{B}^{+},\left\{\pi_{a, b}(\theta): \theta>0\right\}\right) .
$$

It is well known that in this case $F_{x^{\alpha}}(\cdot, \theta)$ is the gamma distribution with shape parameter $\alpha+1$. A trivial verification shows that $\pi_{a, b}$ satisfies conditions (2) and (3). Thus, we can formulate the following theorem.

THEOREM 4. Under the violation $\pi_{a, b}$ of the model $M_{0}$, the uniformly most bias-robust estimate of the scale parameter in the class $\mathcal{T}$ is

$$
T^{0}=\frac{X_{n: n}}{1+\frac{1}{2}+\cdots+\frac{1}{n}} .
$$

Proof. This follows immediately from Theorem 1 in Bartoszewicz (1986). We check at once that

$$
F_{x^{a}}(\cdot ; \theta) \leq_{\text {st }} F(\cdot ; \theta) \leq_{\text {st }} F_{x^{b}}(\cdot ; \theta)
$$

by Theorem 2. It is well known that

$$
F_{x^{b}}(\cdot ; \theta) \leq_{*} F(\cdot ; \theta) \leq_{*} F_{x^{a}}(\cdot ; \theta)
$$

and it suffices to use Lemma 1.

It is easy to see that the above model is an extension of the model considered by Bartoszewicz (1986). 


\section{Robust estimation of the scale parameter in the weighted uniform model}

5.1. Uniform model. Let the original model be $M_{0}=\left(\mathbb{R}^{+}, \mathcal{B}^{+},\{F(\cdot ; \theta)\right.$ : $\theta>0\})$ where $F(\cdot ; \theta)$ is the uniform distribution on $(0, \theta)$. We give an extension of $M_{0}$ under weighting. Define

$$
\pi_{a, b}(\theta)=\left\{F_{w}(\cdot ; \theta): w \in \mathcal{W}_{a, b}\right\}, \quad-1<a<0, b>0 .
$$

Thus we have

$$
M_{1}=\left(\mathbb{R}^{+}, \mathcal{B}^{+},\left\{\pi_{a, b}(\theta): \theta>0\right\}\right) .
$$

We can formulate the following theorem.

THEOREM 5. Under the violation $\pi_{a, b}$ of $M_{0}$, the uniformly most biasrobust estimate of the scale parameter in the class $\mathcal{T}$ is

$$
T^{0}=(n+1) X_{n: n} / n .
$$

Proof. The proof is similar to the proof of Theorem 1.

As previously, this model is an extension of the model considered in Bartoszewicz (1986). Next we give an extension of the uniform model.

5.2. Uniform model with monotone weight function. Now we will consider more general situations than the one considered in Section 5.1.

Let the original model be $M_{0}=\left(\mathbb{R}^{+}, \mathcal{B}^{+},\{F(\cdot ; \theta): \theta>0\}\right)$ where $F(\cdot ; \theta)$ is the uniform distribution on $(0, \theta)$. Let us define the following set of distributions:

$$
\pi_{\mathcal{W}}(\theta)=\left\{F_{w}(\cdot ; \theta): w \in \mathcal{W}\right\}
$$

where $\mathcal{W}$ is the class of positive, monotone weight functions. We obtain an extension of $M_{0}$ :

$$
M_{1}=\left(\mathbb{R}^{+}, \mathcal{B}^{+},\left\{\pi_{\mathcal{W}}(\theta): \theta>0\right\}\right) .
$$

It is obvious that in this case weighted distributions have monotone densities on $[0, \theta]$ and if $w$ is non-decreasing then

$$
F_{w}(\cdot ; \theta) \leq_{\mathrm{c}} F(\cdot ; \theta) \text { and } F(\cdot ; \theta) \leq_{\mathrm{st}} F_{w}(\cdot ; \theta) \text { for every } \theta,
$$

and similarly if $w$ is non-increasing then

$$
F(\cdot ; \theta) \leq_{\mathrm{c}} F_{w}(\cdot ; \theta) \quad \text { and } \quad F_{w}(\cdot ; \theta) \leq_{\mathrm{st}} F(\cdot ; \theta) \quad \text { for every } \theta .
$$

Let $\mathcal{W}^{\prime}$ be the class of functions $w$ which are non-decreasing, and $\mathcal{W}^{\prime \prime}$ the class of functions $w$ which are non-increasing. We check at once that

$$
\begin{aligned}
& \inf _{F_{w}(\cdot ; \theta) \in \pi_{\mathcal{W}}(\theta)} E_{w ; \theta}[T]=\inf _{F_{w}(\cdot ; \theta) \in \pi_{\mathcal{W}^{\prime \prime}}(\theta)} E_{w ; \theta}[T], \\
& \sup _{F_{w}(; ; \theta) \in \pi_{\mathcal{W}}(\theta)} E_{w ; \theta}[T]=\sup _{F_{w}(\cdot ; \theta) \in \pi_{\mathcal{W}^{\prime}}(\theta)} E_{w ; \theta}[T]
\end{aligned}
$$

for any $T \in \mathcal{T}$. We can formulate the following lemma. 
Lemma 3. For any $w^{\prime} \in \mathcal{W}^{\prime}, w^{\prime \prime} \in \mathcal{W}^{\prime \prime}$ and $T \in \mathcal{T}$ we have

$$
E_{w^{\prime} ; \theta}[T]-E_{w^{\prime \prime} ; \theta}[T] \geq \frac{E_{w^{\prime} ; \theta}\left[X_{n: n}\right]-E_{w^{\prime \prime} ; \theta}\left[X_{n: n}\right]}{E_{1}\left[X_{n: n}\right]} .
$$

Proof. It is easy to see from Theorem 1 that for any $w^{\prime}, w^{\prime \prime}$ and $T \in \mathcal{T}$ we have

$$
E_{w^{\prime} ; \theta}[T]-E_{w^{\prime \prime} ; \theta}[T] \geq \min _{1 \leq i \leq n} \frac{E_{w^{\prime} ; \theta}\left[X_{i: n}\right]-E_{w^{\prime \prime} ; \theta}\left[X_{i: n}\right]}{E_{1}\left[X_{i: n}\right]} .
$$

Thus from (5), (6) and Lemma 1 we infer that

$$
\frac{X_{n: n}}{E_{1}\left[X_{n: n}\right]}
$$

minimizes $E_{w^{\prime} ; \theta}[T]-E_{w^{\prime \prime} ; \theta}[T]$ for every $T \in \mathcal{T}$ and every $\theta$.

Now we can formulate the following theorem.

THEOREM 6. Under the violation $\pi_{\mathcal{W}}$ of the model $M_{0}$, the uniformly most bias-robust estimate of the scale parameter in the class $\mathcal{T}$ is

$$
T^{0}=(n+1) X_{n: n} / n .
$$

Proof. This follows from Lemma 3.

6. Estimation of the scale parameter in the weighted exponential model with monotone failure rate. Let the original model be $M_{0}=\left(\mathbb{R}^{+}, \mathcal{B}^{+},\{F(\cdot ; \theta): \theta>0\}\right)$ where $F(\cdot ; \theta)$ is an exponential distribution function with scale parameter $\theta$. Let $\mathcal{W}$ be the class of functions $w$ which are increasing and concave. Assume also that $w(x) \equiv 1$ belongs to $\mathcal{W}$ and for every $w \in \mathcal{W}$ we have

$$
\lim _{x \rightarrow \infty} w(x) e^{-\lambda x}=0, \quad 0<\lambda<1 .
$$

Define

$$
\pi_{\mathcal{W}}(\theta)=\left\{F_{w}(\cdot ; \theta): w \in \mathcal{W}\right\} .
$$

Clearly $\pi_{\mathcal{W}}(\theta)$ satisfies conditions (2) and (3).

Consider the extension of $M_{0}$ under weighting

$$
M_{1}=\left(\mathbb{R}^{+}, \mathcal{B}^{+},\left\{\pi_{\mathcal{W}}(\theta): \theta>0\right\}\right) .
$$

The problem is to find $T^{0} \in \mathcal{T}$, the uniformly most bias-robust estimate of the scale parameter under the violation $\pi_{\mathcal{W}}$. Clearly we have

$$
F(\cdot ; \theta) \leq_{\mathrm{st}} F_{w}(\cdot ; \theta), \quad w \in \mathcal{W},
$$


because $w$ is increasing. Thus we check at once that

$$
\inf _{w \in \mathcal{W}} E_{w ; \theta}[T]=E_{\theta}[T]
$$

for any $T \in \mathcal{T}$.

By definition of IFR distribution and Lemma 2,

$$
F_{w}(\cdot ; \theta) \leq_{\mathrm{c}} F(\cdot ; \theta) \quad \text { for any } w \in \mathcal{W} \text { and every } \theta .
$$

We can formulate the following lemma similar to Lemma 3 , and a theorem similar to Theorem 6 .

Lemma 4. For any $w \in \mathcal{W}$ and $T \in \mathcal{T}$ we have

$$
E_{w ; \theta}[T]-E_{\theta}[T] \geq \frac{E_{w ; \theta}\left[X_{n: n}\right]-E_{\theta}\left[X_{n: n}\right]}{E_{1}\left[X_{n: n}\right]} .
$$

THEOREM 7. Under the violation $\pi_{\mathcal{W}}$ of the model $M_{0}$ the uniformly most bias-robust estimate of the scale parameter is

$$
T^{0}=\frac{X_{n: n}}{1+\frac{1}{2}+\cdots+\frac{1}{n}} .
$$

Proof. This follows from (7), (8) and Lemma 4.

\section{References}

B. C. Arnold (1980), Majorization and the Lorenz Order: A Brief Introduction, Lecture Notes in Statist. 43, Springer.

R. E. Barlow and F. Proschan (1966), Inequalities for linear combinations of order statistics from restricted families, Ann. Math. Statist. 37, 1574-1592.

J. Bartoszewicz (1986), Bias-robust estimation of the scale parameter, Probab. Math. Statist. 7, 103-113.

J. Bartoszewicz and M. Skolimowska (2006), Preservation of classes of life distributions and stochastic orders under weighting, Statist. Probab. Lett. 76, 587-596.

M. J. Bayarri and J. Berger (1998), Robust bayesian analysis of selection models, Ann. Statist. 26, 645-659.

Y. Chung and C. Kim (2004), Measuring robustness for weighted distributions: Bayesian perspective, Statist. Papers 45, 15-31.

R. A. Fisher (1934), The effects of methods of ascertainment upon the estimation of frequencies, Ann. Eugenics 6, 13-25.

K. Jain, H. Singh and I. Bagai (1989), Relations for the reliability measures of weighted distributions, Comm. Statist. Theory Methods 18, 4393-4412.

G. P. Patil and J. K. Ord (1976), On size-biased sampling and related form invariant weighted distributions, Sankhyā B 38, 48-61.

G. P. Patil and C. R. Rao (1977), The weighted distributions: A survey and their applications, in: Applications of Statistics, P. R. Krishnaiah (ed.), North-Holland, Amsterdam, 383-405.

C. R. Rao (1985), Weighted distributions arising out of methods of ascertainment: What population does a sample represent?, in: Celebration of Statistics: The ISI Centenary Volume, A. C. Atkinson and S. E. Fienberg (eds.), Springer, New York, 543-569. 
R. Zieliński (1977), Robustness: a quantitative approach, Bull. Acad. Polon. Sci. Sér. Sci. Math. Astronom. Phys. 25, 1281-1286.

R. Zieliński (1983), A most bias-robust linear estimate of the scale parameter of the exponential distribution, Appl. Math. (Warsaw) 18, 73-77.

Mathematical Institute

University of Wrocław

Pl. Grunwaldzki 2/4

50-384 Wrocław, Poland

E-mail: blazej@math.uni.wroc.pl

Received on 6.12.2006;

revised version on 8.3.2007 